Nanotechnology

\title{
Elastic Properties of Photoswitchable Azobenzene Polymers from Molecular Dynamics Simulations**
}

\author{
Lars V. Schäfer, E. Matthias Müller, Hermann E. Gaub, and Helmut Grubmüller*
}

Nanomechanical devices or molecular machines will, for a broad range of applications, most likely be powered by light or other kinds of electromagnetic radiation. ${ }^{[1-4]}$ The major reasons are ease of addressability, picosecond reaction times to external stimuli, and compatibility with a broad range of ambient substances, such as solvents, electrolytes, or gases. Azobenzene is a well-studied photoactive system, which can be photoswitched selectively from an extended trans to a more compact cis conformation by using light of wavelength $365 \mathrm{~nm}$, whereas the reverse cis-to-trans isomerization is induced by light of wavelength $420 \mathrm{~nm}$ (Figure 1a). ${ }^{[5,6]}$ Many processes, such as light-driven ion transport through biological membranes, ${ }^{[7-9]}$ can be steered by conformational switching of azobenzene chromophores. Azobenzene has been used frequently in synthetic photoresponsive systems for the regulation of the geometry and function of biomolecules. ${ }^{[10-19]}$ The isomerization of individual azobenzene molecules has also been studied by scanning tunneling microscopy. ${ }^{[20,21]}$

Recently, a bistable polyazobenzene peptide was synthesized as a model system for a light-powered molecular machine, and its mechanical properties were characterized by means of single-molecule atomic force microscopy (AFM) experiments (Figure 1b,c). ${ }^{[22,23]}$ Optical switching of the azobenzene polymers between their extended trans and compact cis conformations was demonstrated, and the corresponding change in the contour length of the polymer was detected. Thereby, in analogy to an Otto cycle, Gaub and co-workers established an optomechanical operating cycle, in which "optical" contraction against an external force delivered net mechanical work. ${ }^{[22,23]}$ Thus, they demonstrated that azobenzene polymers indeed hold great promise for future

[*] L. V. Schäfer, E. M. Müller, H. Grubmüller Abteilung für theoretische und computergestützte Biophysik Max-Planck-Institut für Biophysikalische Chemie

Am Fassberg 11, 37077 Göttingen (Germany)

Fax: (+49) 551-201-2302

E-mail: hgrubmu@gwdg.de

H. E. Gaub

Lehrstuhl für Angewandte Physik und Center of Nanoscience Ludwig-Maximilians-Universität

Amalienstrasse 54, 80799 München (Germany)

[***] We thank I. Tremmel for carefully reading the manuscript and for valuable comments, and F. Gräter, O. Lange, R. Netz, D. Oesterhelt, and C. Renner for helpful discussions. L.V.S. thanks the Boehringer Ingelheim Fonds for a PhD fellowship. Support from the EU Nanomot project (grant 29084) is gratefully acknowledged. H.E.G. was supported by the Deutsche Forschungsgemeinschaft and the Fonds der Chemischen Industrie.

0 Supporting information for this article is available on the WWW under http://www.angewandte.org or from the author. applications in nanotechnology, for example, as light-triggered molecular switches or cargo lifters. In a related experiment, Vancso and co-workers characterized a redoxmechanical cycle by using electrochemical AFM-based single-molecule force spectroscopy. ${ }^{[24]}$

a)
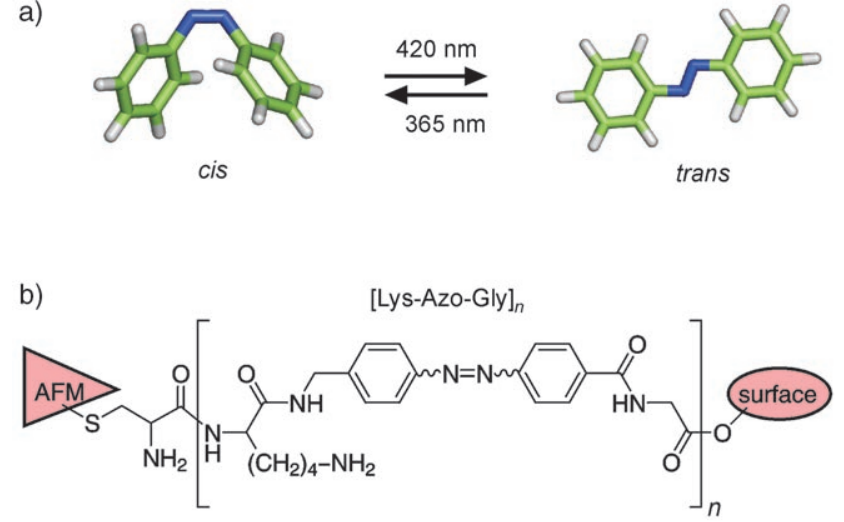

c)

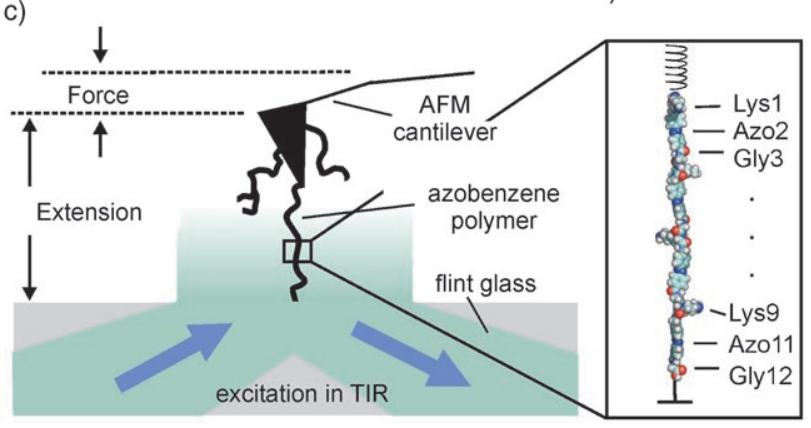

Figure 1. a) Photochromic azobenzene can be switched between a compact cis and an extended trans conformation. b) A polyazobenzene peptide composed of Lys-Azo-Gly azotripeptide units. c) Sketch of the AFM experiment which motivated the study described herein. ${ }^{[22,23]}$ The azobenzene polymer was attached to the AFM tip through a cysteine linker (top) and mounted to an amino-functionalized surface (bottom); adapted with permission from the authors of ref. [22]. $T I R=$ total internal reflection. d) Space-filling representation of the alltrans conformation of the model polymer used for the force-probe MD simulations. To mimic the AFM experiments, a harmonic spring was attached to the $\mathrm{N}$ terminus (top) and pulled upwards with a constant velocity, while the $\mathrm{C}$ terminus was kept fixed (bottom).

However, the measured overall length change upon switching between the cis and trans polymers-and thus the work output-was considerably smaller than could be expected on the basis of the length change of a single 
azobenzene monomer and the number of individual monomers. To resolve this discrepancy, and, more generally, to obtain a detailed understanding of the underlying polymer mechanics at the atomistic scale, we carried out explicitsolvent force-probe molecular dynamics (MD) simulations ${ }^{[25,26]}$ of polyazobenzene model peptides under mechanical stress, the results of which resemble those of the AFM experiments described in Figure $1 \mathrm{c}$. The simulations provided detailed mechanistic insight that is prerequisite for the efficient and targeted optimization of photoswitchable polymers for future applications in nanotechnology. Because we were only interested in the elastic properties of these model polymers, and not in the photoisomerization kinetics, we kept the azobenzenes fixed in the cis or trans conformation during the simulations. We did not include the photoisomerization itself into our simulations on the assumption that this process occurs on a much faster time scale than the subsequent structural rearrangement of the polymer. Indeed, sub-picosecond kinetics have been observed for azobenzene photoisomerization, ${ }^{[27,28]}$ a complex process that involves several energy surfaces. ${ }^{[27-33]}$

By comparing our simulations to the AFM experiments, we gleaned insight into the overall elastic properties of azobenzene polymers and could relate our results directly to the measured elasticity characteristics. We were able to elucidate the crucial role of the peptides that interlink the azobenzene units and to predict the elastic behavior beyond the experimentally accessible force regime. Finally, we used the detailed mechanistic understanding thus obtained to design a photoswitchable polyazobenzene peptide with improved optomechanical properties.

The model azobenzene polymers used in our simulations were constructed from four azotripeptide units $(n=4$ in Figure $1 \mathrm{~b}$ ). These (Lys-Azo-Gly) ${ }_{4}$ dodecamers have the same stoichiometry as the polymers used in the AFM studies. As in the experiments, DMSO was used as a solvent. To study the effect of the azobenzene conformation (cis or trans) on the elastic properties, we considered three stereochemically different polymers, an all-cis, an all-trans, and a mixed cistrans-cis-trans system. The mixed polymer was considered because complete photoisomerization upon optical pumping was not achieved in the experiments as a result of the overlapping absorption bands of the cis and trans states. ${ }^{[22,23]}$ A study of this polymer would also reveal possible cooperative effects between adjacent monomers.

The force-versus-extension traces obtained from the AFM experiments reflect the elastic properties expected for a polymer subjected to a mechanical load. Figure 2 a shows the overall force-extension traces of the all-cis, all-trans, and mixed azobenzene model polymers obtained from our forceprobe MD simulations (over 50-70 ns each). These traces closely resemble the curves from the AFM experiments (Figure 2a, inset). ${ }^{[22,23]}$ At higher forces above $600 \mathrm{pN}$, deviations from the wormlike-chain (WLC) behavior are seen. These deviations are discussed below. As expected, the force trace of the mixed cis-trans-cis-trans polymer lies midway between those of the all-cis and the all-trans polymers. This result already suggests that cooperative effects between monomers are unlikely. Therefore, such effects cannot
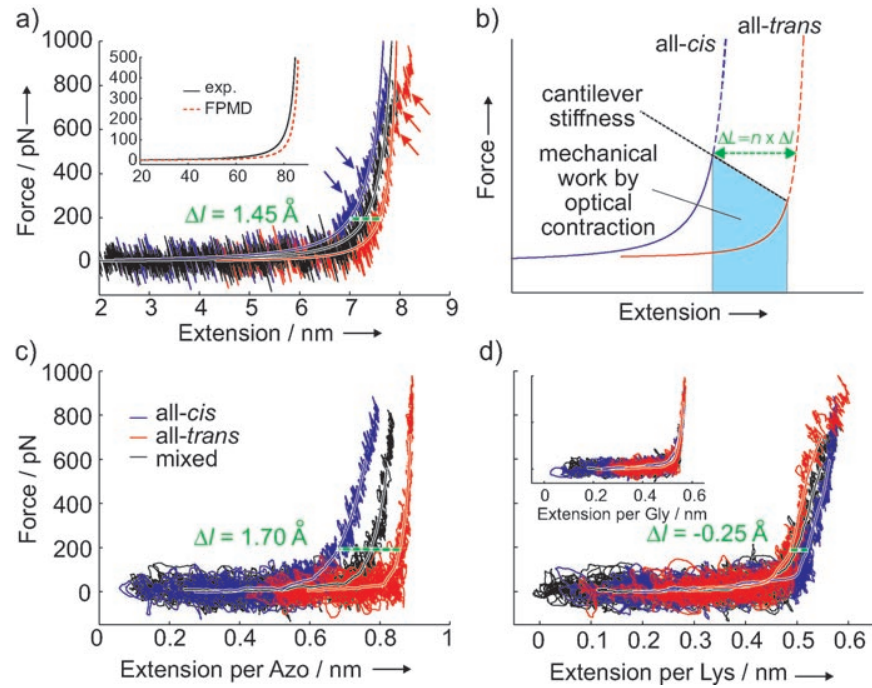

Figure 2. Force-probe MD force-extension curves for (Lys-Azo-Gly) ${ }_{4}$, obtained at a pulling velocity of $0.1 \mathrm{~nm} \mathrm{~ns}^{-1}$. a) Curves of the all-cis (blue), all-trans (red), and mixed cis-trans-cis-trans polymers (black). The overall extension is defined as the distance between the $C$ terminus and the $\mathrm{N}$ terminus along the pulling direction. The extension difference obtained from a WLC fit (smooth lines) of $1.45 \AA$ per unit between the cis and trans polymers (at $200 \mathrm{pN}$ ) is shown as a green dashed line. Arrows indicate events discussed in the text. Inset: WLC curves obtained from experiment (black line) ${ }^{[23]}$ and simulation (red dashed line) for the all-trans polymer, for the contour length of $L_{c}=89.1 \mathrm{~nm}$ taken from ref. [23]. b) The output of mechanical work (blue trapezoid) due to "optical" contraction against an external load increases with the extension difference, $\Delta L$, which is the extension difference per monomer, $\Delta l$, multiplied by the number of monomers, $n$. The cantilever stiffness is reflected in the slope of the dashed black line. c, d) Force-extension curves of the individual polymer building blocks azobenzene (c), lysine (d), and glycine (d, inset). The smooth lines represent 100000 -point averages. For the definition of the boundaries of the individual monomers, see the Supporting Information.

explain the unexpectedly small length changes observed in the AFM experiments.

The force field and simulation protocol used were validated by comparison with the AFM results. To this end, the force-probe MD force-extension data were modeled using an extended WLC model, ${ }^{[34]}$ WLC model [Eq. (1)], ${ }^{[34]}$

$F(r)=\frac{k_{\mathrm{B}} T}{L_{\mathrm{p}}}\left(\frac{1}{4}\left(1-\frac{r}{L_{\mathrm{c}}}\right)^{-2}-\frac{1}{4}+\frac{r}{L_{\mathrm{c}}}\right)$,

in which $F$ is the force, $T=300 K$ the temperature, $r$ the polymer extension, $L_{\mathrm{c}}$ the contour length, and $L_{\mathrm{p}}$ the persistence length. Table 1 lists the WLC parameters obtained as well as the extension difference per switched azobenzene monomer, $\Delta l$, for all simulated systems, together with the values obtained from experiment.

Because experimental WLC data are only accessible for the all-trans polymer, ${ }^{[23]}$ we compare in Table 1 only the parameters of the all-trans polymers. The contour length $L_{\mathrm{c}}$ per azotripeptide unit given in reference [22] was not extracted from the measured force traces, but determined by molecular modeling. Therefore, we only compare the 
persistence lengths $L_{\mathrm{p}}$. These lengths obtained from the simulations range from $0.40 \mathrm{~nm}$ to $0.97 \mathrm{~nm}$ and agree with the experimental value of $0.5 \mathrm{~nm}$ (Table 1 ) within our statistical accuracy. We found $L_{\mathrm{p}}\left(R_{\text {free }}^{2}\right)$ to be quite sensitive to the details of the fit. The quality of the fit did not drop significantly when the experimental value of $L_{\mathrm{p}}=0.5 \mathrm{~nm}$ was used $\left(R_{\mathrm{fix}}^{2}\right.$ in Table 1$)$. This good agreement between theory and experiment validates the force field applied and renders finite size effects of the dodecamers used as model systems for the more extended azobenzene polymers used in the AFM experiments unlikely. Further tests to confirm the validity of our approach are presented in the Supporting Information.

Table 1: WLC parameters of all-trans polymers obtained from experiment and from our force-probe MD simulations. ${ }^{[a]}$

\begin{tabular}{llllll}
\hline & $\begin{array}{l}\Delta / / \text { mono- } \\
\operatorname{mer}[\AA]\end{array}$ & $\begin{array}{l}L_{c} / \text { mono- } \\
\operatorname{mer}[\mathrm{nm}]\end{array}$ & $L_{\mathrm{p}}[\mathrm{nm}]$ & $R_{\text {free }}^{2}$ & $R_{\text {fix }}^{2}$ \\
\hline experiment $^{[22]}$ & 0.60 & 1.90 & 0.50 & & \\
simulation $^{2}$ & 1.45 & 2.05 & 0.97 & 0.88 & 0.85 \\
\hline
\end{tabular}

[a] WLC parameters were obtained by modeling the force-extension data with the extended WLC model [Eq. (1)]. The extension difference $\Delta /$ per switched azobenzene monomer is given at a force of $200 \mathrm{pN}$ to enable direct comparison with the experimental data. Two different values are given for $R^{2}$; these values reflect the quality of the fits. $R_{\text {free }}^{2}$ was obtained from a WLC fit with both $L_{c}$ and $L_{p}$ as adjustable parameters, whereas $R_{\text {fix }}^{2}$ was obtained from a fit with $L_{p}=0.5 \mathrm{~nm}$, which is the value obtained from the experimental data.

The WLC fits were also used to extract the extension differences $\Delta l$ between the trans and cis polymers from the simulations. As illustrated in Figure $2 \mathrm{~b}, \Delta l$ determines the work output upon contraction against an external load. Therefore, an increase in $\Delta l$ improves the efficiency of the optomechanical energy conversion caused by the polymers.

The extension difference between the cis and trans states of an isolated azobenzene molecule was estimated from quantum-chemical calculations to be $2.5 \AA{ }^{\left[{ }^{[29]}\right.}$ However, the polymer typically does not undergo the corresponding length change, because at low forces $(F<200 \mathrm{pN})$ the azobenzene monomers are not aligned perfectly, and thus only part of the cis-trans length change of the monomer is available in the pulling direction. In contrast, at higher forces the polymers are aligned to a larger extent, and thus could undergo larger length changes. However, trans-azobenzene is considerably stiffer than cis-azobenzene (see the Supporting Information), which again reduces the observed length change. From our simulations, in which these effects were taken fully into account, we predict an extension difference of about 1.3 to $1.5 \AA$ per switched azobenzene unit. As the measured extension difference of about $0.6 \AA$ is still smaller by more than a factor of two (Table 1), the work output might be improved. Closer inspection shows that only part of this reduction in polymer-length change, $\Delta l$, can be explained by the above two effects.

To identify the origin of the as yet unexplained reduction in $\Delta l$, we decomposed the overall force-extension curves into the contributions of the individual polymer building blocks.
Indeed, the extension difference due solely to the azobenzene units is $1.70 \AA$ per monomer (dashed green line in Figure 2c), which is the value expected from polymer tilt and differential stiffness. This contribution to $\Delta l$ is larger than the total extension difference of $1.45 \AA$ for the polymer (Figure $2 \mathrm{a}$ ). Figure $2 \mathrm{~d}$ shows that the difference of about $0.25 \AA$ can be attributed to the lysine residues, which therefore partly compensate the "optical" lengthening and contraction of the azo groups. The force-extension curves of the glycine residues are rather independent of the azobenzene conformation (Figure $2 \mathrm{~d}$, inset).

As shown schematically on the right-hand side of Figure $3 \mathrm{~d}$, the compensation by lysine residues is due to the isomerization of the lysine backbone between an extended anti and a more compact syn conformation around the backbone dihedral angle $\Psi$. These two conformers are populated to different extents, depending on whether the
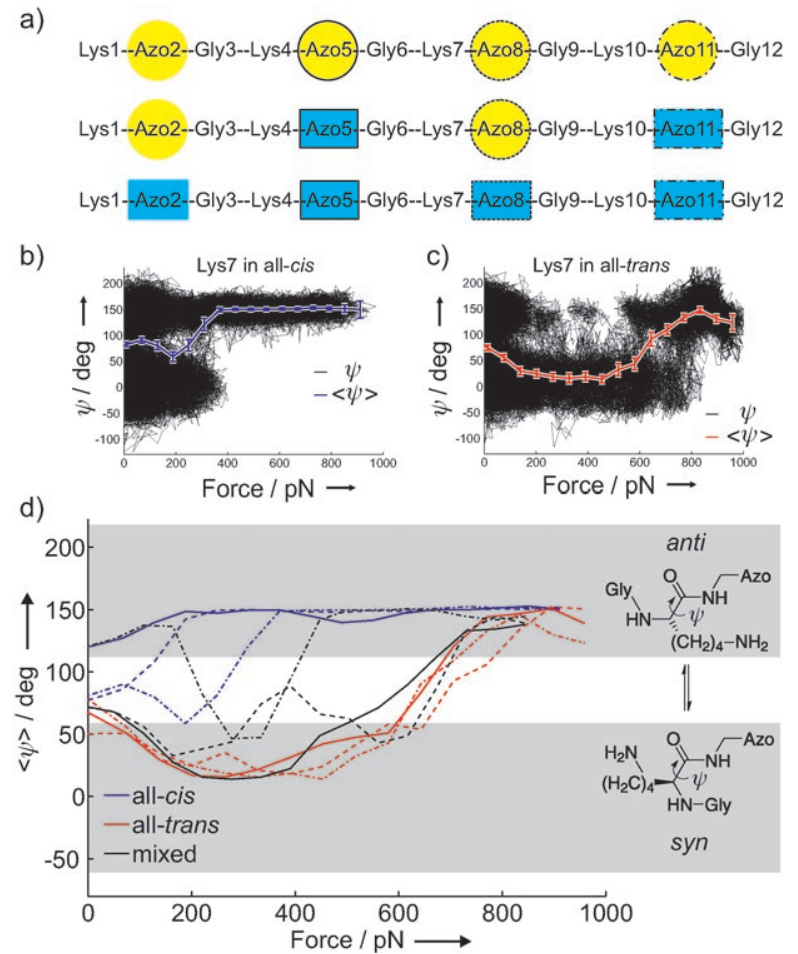

Figure 3. Syn-anti conformational switching of lysine amino acids. a) Sketch of the all-cis (top), mixed (middle), and all-trans polymers (bottom). Azobenzene units in the cis conformation are shown as yellow circles, those in the trans conformation as turquoise rectangles. b, c) Exemplarily, the backbone dihedral angles $\Psi$ of Lys 7 in the all-cis and all-trans polymers are plotted as a function of the applied force. Averages of $\Psi$ were calculated with a 50-pN window. Error bars represent the errors of the mean and are also typical for the data shown in panel (d). d) Extended anti and compact syn lysine conformations interconvert by rotation about the backbone dihedral angle $\Psi$ (right). Averaged $\Psi$ angles of all individual lysine monomers are plotted as a function of the applied force for the all-cis (blue), all-trans (red), and mixed polymers (black) for Lys4 (solid), Lys7 (dash-dotted), and Lys 10 (dashed line). All forces were obtained at a pulling velocity of $0.1 \mathrm{~nm} \mathrm{~ns}^{-1}$. The areas shaded in gray indicate the extended anti and the more compact syn conformations. Lys 1 is directly attached to the harmonic pulling potential (see Figure 1d) and was therefore excluded from the analysis. 
adjacent azobenzene moiety is cis or trans (Figure 3). Figure $3 \mathrm{~b}$, c shows exemplarily the backbone dihedral angles $\Psi$ of Lys7 in the all-cis and in the all-trans polymers, respectively, as a function of the applied force (black), together with the average $\Psi$ angle and the statistical error (blue and red for cis and trans, respectively). In Figure $3 \mathrm{~d}$, the average backbone dihedral angle $\Psi$ of all lysine residues is shown as a function of the applied force. For the all-cis polymer, the lysine residues already adopted the extended anti conformation at very low forces of between 0 and $300 \mathrm{pN}$ (blue lines). These transitions also reveal themselves as steps in the force-extension trace of the all-cis model polymer (blue arrows in Figure 2).

In the mixed polymer, Lys7, which is bonded directly to a cis azobenzene moiety (Figure $3 \mathrm{a}$ ), also adopted the anti conformation very early (dash-dotted black line in Figure $3 \mathrm{~d}$ ), in a similar manner to Lys4 in the all-cis polymer. In contrast, Lys 4 and Lys10 with adjacent trans azobenzene units underwent syn-to-anti transitions only at rather large forces of between 600 and $800 \mathrm{pN}$ (solid and dashed black lines, respectively). The same behavior was observed for all lysine amino acids in the all-trans polymer (red lines).

In summary, our simulations showed that lysine residues bonded to trans azobenzene moieties tend to adopt the compact syn conformation (at up to $600-800 \mathrm{pN}$ ), whereas lysine residues adjacent to cis azo groups already undergo syn-to-anti conformational transitions at low to moderate forces below $300 \mathrm{pN}$. This preference for the extended anti conformation of lysine residues adjacent to cis azobenzene units explains the observed compensation effect. The difference in the length of the polymer chain because of cis-trans isomerization of the azobenzene units is reduced from $1.7 \AA$ to $1.3-1.5 \AA$. However, in the AFM experiments, a value of only $0.6 \AA$ was measured. We conclude that in the AFM experiments only about $40 \%$ of the azobenzene moieties were effectively photoisomerized upon optical pumping. The major reason for the failure to attain the maximum value, which is about $70-80 \%$ as a result of the overlapping absorption bands of the trans and cis isomers, ${ }^{[35]}$ in the AFM experiments is probably that only about half of the polymer was excited by the evanescent light field, in agreement with the estimate of the authors. ${ }^{[22,23,36]}$

The force-probe MD simulations also allow the investigation of the mechanics of the polymers at high forces and thus enable prediction of their elastic behavior even well beyond the force regime accessible to AFM. Only at forces larger than $1000 \mathrm{pN}$ were mechanically induced cis-to-trans transitions of the azobenzene units observed in our simulations (data not shown). These isomerizations occurred by rotation about the central $\mathrm{N}=\mathrm{N}$ bond, in agreement with recent $\mathrm{ab}$ initio calculations. ${ }^{[36]}$ The high forces observed are consistent with the large thermal barrier to isomerization of $108 \mathrm{~kJ} \mathrm{~mol}^{-1},{ }^{[37]}$ which, as a result of the orthogonality of the rotation coordinate with respect to the pulling coordinate, is largely unaffected by the applied force. ${ }^{[36]}$ Also in the AFM experiments no mechanical transitions were observed up to $500 \mathrm{pN}^{[22,23]}$ At large forces of about $600-800 \mathrm{pN}$, the simulations also predict syn-to-anti transitions of the lysine residues in the all-trans polymer. For the short polymer used in this study, these transitions are visible as steps in the forceextension curve (red arrows in Figure 2a). For the much larger polymers used in the AFM experiments, these individual steps would not be resolved, but a tendency towards larger extensions would be observed in the force-extension traces of the trans polymers at forces larger than about $600 \mathrm{pN}$.

The dependence of the degree of extension of the lysine amino acids on the conformation of the azobenzene moiety they are bonded to demonstrates that the peptides interlinking the photoactive azobenzenes are not simply "passive glue", but rather, through intermonomeric correlations, actively affect the elastic properties of the polymer. In the case at hand, the compensating effect of the lysine residues reduces the overall "optical" length contraction and thereby diminishes the work output of the polymer. We next investigated the possibility of using the structural and dynamical insight obtained from our studies to tailor and optimize photoswitchable polymers. For example, with the aim to increase the rigidity of the polymer backbone, we replaced the lysine and glycine residues with proline residues, so that less compensation and, accordingly, a larger work output could be expected.

Figure 4 shows the elastic behavior of the all-cis, all-trans, and mixed (Pro-Azo-Pro) ${ }_{4}$ dodecamers as determined from a set of force-probe MD simulations similar to those described above. The stiffer proline linkers indeed lead to an increased
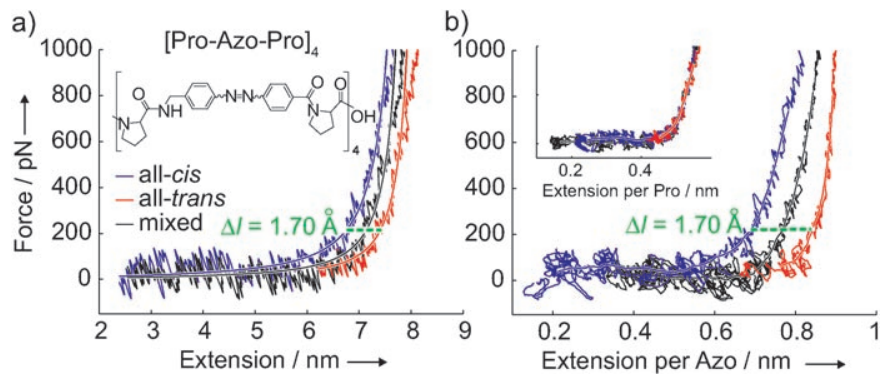

Figure 4. Force-probe MD force-extension curves obtained at a pulling velocity of $0.5 \mathrm{~nm} \mathrm{~ns}^{-1}$ for the (Pro-Azo-Pro) ${ }_{4}$ polymer. a) Curves of the whole all-cis (blue), all-trans (red), and mixed cis-trans-cis-trans polymers (black). Smooth lines describe the WLC fits. The extension difference between the cis and trans polymers is $1.70 \AA$ / unit at $200 \mathrm{pN}$ (dashed green line). b) Force-extension curves of individual azobenzene units and of proline residues (inset). The smooth lines represent 20000-point averages.

extension difference between the cis and trans polymers of $1.70 \AA$ per azo unit (dashed green line), as compared to $1.45 \AA$ for the lysine-containing polymer (see Figure 2). Figure $4 \mathrm{~b}$ shows the individual force-extension curves of the azobenzene and proline moieties in (Pro-Azo-Pro) $)_{4}$. Apparently, and in contrast to (Lys-Azo-Gly), the extension difference between the cis and trans azobenzene units is no longer compensated by the interlinking residues, which explains the increased $\Delta l$ value of $1.70 \AA$. Accordingly, the work output is expected to be enhanced by about $15-20 \%$ relative to that of the lysine-containing polymer.

Efficient and targeted optimization of photoswitchable polymers is a major challenge and requires detailed microscopic interpretation of the polymer mechanics. Herein, we 
provided insight on the basis of force-probe molecular dynamics simulations into the mechanics of photoswitchable polyazobenzene peptides under mechanical stress. Comparison of the WLC parameters revealed that the overall elastic properties of the simulated azobenzene model polymers agree very well with the results of AFM experiments. ${ }^{[22,23]}$ Our simulations showed that the maximal obtainable extension difference between the cis and trans conformations is about $1.7 \AA$ per switched azobenzene unit. However, in the AFM experiments, a value of only $0.6 \AA$ was measured. By decomposing the force versus extension traces into the contributions of the individual building blocks, we identified the interlinking lysine amino acids as one reason for this discrepancy. The lysine linkers partly compensate the contraction and lengthening of the polymer upon optical cis-trans switching of the azobenzene units to 1.3-1.5 $\AA$ and thus are promising targets for optimization. As an example, we constructed an optimized polyazobenzene peptide with stiffer proline linkers, which indeed led to a larger extension difference between the cis and trans forms and thus should enhance the work output by about $15-20 \%$.

From a more general perspective, to ensure the proper functioning of a machine composed of force-generating units and interlinking units, the linkers have to be constructed in such a way as to sustain the mechanical stress generated. We have demonstrated in detail that this principle also holds for machines at the molecular level, such as photoswitchable azobenzene polymers.

\section{Methods}

All simulations were carried out with the Gromacs simulation suite ${ }^{[38]}$ by using the OPLS all-atom force field ${ }^{[39]}$ and periodic boundary conditions. $N p T$ ensembles were simulated with the polymer and solvent coupled separately to a heat bath at $300 \mathrm{~K}\left(\tau_{\mathrm{T}}=0.1 \mathrm{ps}\right) \cdot{ }^{[40]}$ The systems were coupled isotropically to a pressure bath at 1 bar $\left(\tau_{\mathrm{p}}=\right.$ $1.0 \mathrm{ps}) .^{[40]}$ Application of the Lincs ${ }^{[41]}$ algorithm allowed an integration time step of $2 \mathrm{fs}$. Short-range electrostatic and Lennard-Jones interactions were calculated within a cutoff of $1.0 \mathrm{~nm}$ and $1.4 \mathrm{~nm}$, respectively. The particle mesh Ewald (PME) method was used for the long-range electrostatic interactions. ${ }^{[42]}$ Depending on the capping of the lysine side chains and the azobenzene conformations, total system sizes were between 35000 and 57000 atoms. During the force-probe simulations, the $\mathrm{N}$ terminus of the polymer, $\mathrm{N}^{\alpha}$, was subjected to a moving harmonic spring potential [Eq. (2)],

$V_{\text {spring, } \mathrm{N}^{a}}(t)=k_{0}\left[z_{\mathrm{N}^{a}}(t)-z_{\text {spring }}(t)\right]^{2}$

in which $k_{0}=500 \mathrm{~kJ} \mathrm{~mol}^{-1} \mathrm{~nm}^{-2}$ is the force constant of the spring, $z_{\mathrm{N}^{\alpha}}(t)$ is the position of the pulled $\mathrm{N}^{\alpha}$ atom, and $z_{\text {spring }}(t)$ the position of the spring. The $\mathrm{C}$ terminus, $\mathrm{C}^{\omega}$, was fixed by a stationary harmonic potential $\left(k_{\mathrm{fix}}=1000 \mathrm{~kJ} \mathrm{~mol}^{-1} \mathrm{~nm}^{-2}\right)$. Mechanical stress to probe the elastic behavior of the model polymers was applied by moving the spring with a constant velocity $v$ in the positive $z$ direction, $z_{\text {spring }}(t)=$ $z(0)+v t$, with $z(0)=z_{\mathbb{N}^{a}}(0)$. Force-probe MD simulations were carried out with pulling velocities of $v=0.1$ and $0.5 \mathrm{~nm} \mathrm{~ns}^{-1}$ to yield simulation times of 50-70 and 10-14 ns, respectively, per force-probe MD trajectory. The overall simulation time was about $0.5 \mu \mathrm{s}$. Simulation details are given in the Supporting Information.

Received: November 10, 2006

Published online: February 15, 2007
Keywords: azo compounds - molecular dynamics . nanotechnology $\cdot$ polymers $\cdot$ scanning probe microscopy

[1] J. M. Lehn, Supramolecular Chemistry: Concepts and Perspectives, Wiley-VCH, Weinheim, 1995.

[2] J. P. Collin, P. Gavina, V. Heitz, J. P. Sauvage, Eur. J. Inorg. Chem. 1998, 1.

[3] V. Balzani, A. Credi, F. M. Raymo, J. F. Stoddart, Angew. Chem. 2000, 112, 3484; Angew. Chem. Int. Ed. 2000, 39, 3348.

[4] B. L. Feringa, R. A. van Delden, N. Koumura, E. M. Geertsema, Chem. Rev. 2000, 100, 1789.

[5] G. S. Hartley, Nature 1937, 140, 281.

[6] H. Dürr, H. B. H. Rau, Photochromism: Molecules and Systems, Elsevier, Amsterdam, 1990, chap. 4.

[7] S. Shinkai, O. Manabe, Top. Curr. Chem. 1984, 121, 67.

[8] R. H. Kramer, J. J. Chambers, D. Trauner, Nat. Chem. Biol. 2005, 1,360 .

[9] H. Bayley, Nat. Chem. Biol. 2006, 2, 11.

[10] L. Ulysse, J. Cubillos, J. Chmielewski, J. Am. Chem. Soc. 1995, 117,8466 .

[11] I. Willner, Acc. Chem. Res. 1997, 30, 347.

[12] H. Asanuma, T. Ito, T. Yoshida, X. G. Liang, M. Komiyama, Angew. Chem. 1999, 111, 2547; Angew. Chem. Int. Ed. 1999, 38, 2393.

[13] S. Spörlein, H. Carstens, H. Satzger, C. Renner, R. Behrendt, L. Moroder, P. Tavan, W. Zinth, J. Wachtveitl, Proc. Natl. Acad. Sci. USA 2002, 99, 7998.

[14] V. Kräutler, A. Aemissegger, P. H. Hünenberger, D. Hilvert, T. Hansson, W. F. van Gunsteren, J. Am. Chem. Soc. 2005, 127, 4935.

[15] A. Aemissegger, V. Kräutler, W. F. van Gunsteren, D. Hilvert, J. Am. Chem. Soc. 2005, 127, 2929.

[16] L. Guerrero, O. S. Smart, C. J. Weston, D. C. Burns, G. A. Woolley, R. K. Allemann, Angew. Chem. 2005, 117, 7956; Angew. Chem. Int. Ed. 2005, 44, 7778.

[17] J. Bredenbeck, J. Helbing, J. R. Kumita, G. A. Woolley, P. Hamm, Proc. Natl. Acad. Sci. USA 2005, 102, 2379.

[18] P. H. Nguyen, Y. G. Mu, G. Stock, Proteins Struct. Funct. Genet. 2005, 60, 485 .

[19] P. H. Nguyen, G. Stock, Chem. Phys. 2006, 323, 36.

[20] S. Yasuda, T. Nakamura, M. Matsumoto, H. Shigekawa, J. Am. Chem. Soc. 2003, 125, 16430

[21] J. Henzl, M. Mehlhorn, H. Gawronski, K. H. Rieder, K. Morgenstern, Angew. Chem. 2006, 118, 617; Angew. Chem. Int. Ed. 2006, 45, 603 .

[22] T. Hugel, N. B. Holland, A. Cattani, L. Moroder, M. Seitz, H. E. Gaub, Science 2002, 296, 1103.

[23] N. B. Holland, T. Hugel, G. Neuert, A. Cattani-Scholz, C. Renner, D. Oesterhelt, L. Moroder, M. Seitz, H. E. Gaub, Macromolecules 2003, 36, 2015.

[24] S. Zou, M. A. Hempenius, H. Schönherr, G. J. Vancso, Macromol. Rapid Commun. 2006, 27, 103.

[25] H. Grubmüller, B. Heymann, P. Tavan, Science 1996, $271,997$.

[26] B. Isralewitz, S. Izrailev, K. Schulten, Biophys. J. 1997, 73, 2972.

[27] T. Schultz, J. Quenneville, B. Levine, A. Toniolo, T. J. Martinez, S. Lochbrunner, M. Schmitt, J. P. Shaffer, M. Z. Zgierski, A. Stolow, J. Am. Chem. Soc. 2003, 125, 8098.

[28] C. Nonnenberg, H. E. Gaub, I. Frank, ChemPhysChem 2006, 7, 1455.

[29] S. Monti, G. Orlandi, P. Palmieri, Chem. Phys. 1982, 71, 87.

[30] P. Cattaneo, M. Persico, Phys. Chem. Chem. Phys. 1999, 1, 4739.

[31] C. Ciminelli, G. Granucci, M. Persico, Chem. Eur. J. 2004, 10, 2327.

[32] A. Cembran, F. Bernardi, M. Garavelli, L. Gagliardi, G. Orlandi, J. Am. Chem. Soc. 2004, 126, 3234

[33] C. R. Crecca, A. E. Roitberg, J. Phys. Chem. A 2006, 110, 8188. 
[34] C. Bustamante, J. F. Marko, E. D. Siggia, S. Smith, Science 1994 $265,1599$.

[35] R. Behrendt, C. Renner, M. Schenk, F. Q. Wang, J. Wachtveitl, D. Oesterhelt, L. Moroder, Angew. Chem. 1999, 111, 2941; Angew. Chem. Int. Ed. 1999, 38, 2771.

[36] G. Neuert, T. Hugel, R. R. Netz, H. E. Gaub, Macromolecules 2006, 39, 789 .

[37] A. A. Blevins, G. J. Blanchard, J. Phys. Chem. B 2004, 108, 4962.
[38] D. van der Spoel, B. Hess, E. Lindahl, G. Groenhof, A. E. Mark, H. J. C. Berendsen, J. Comput. Chem. 2005, 26, 1701.

[39] W. L. Jorgensen, J. Tirado-Rives, J. Am. Chem. Soc. 1988, 110, 1657.

[40] H. J. C. Berendsen, J. P. M. Postma, W. F. van Gunsteren, A. DiNola, J. R. Haak, J. Chem. Phys. 1984, 81, 3684.

[41] B. Hess, H. Bekker, H. J. C. Berendsen, J. G. E. M. Fraaije, J. Comput. Chem. 1997, 18, 1463.

[42] T. Darden, D. York, L. Pedersen, J. Chem. Phys. 1993, 98, 10089. 